\title{
Sauerwein, Markus
}

\section{Qualitätsmerkmale in Ganztagsangeboten aus Perspektive der Schüler/innen}

formal und inhaltlich überarbeitete Version der Originalveröffentlichung in:

formally and content revised edition of the original source in:

Schulpädagogik heute 7 (2016) 13, 21 S.

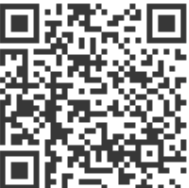

Bitte verwenden Sie in der Quellenangabe folgende URN oder DOI /

Please use the following URN or DOI for reference:

urn:nbn:de:0111-pedocs-193299

10.25656/01:19329

https://nbn-resolving.org/urn:nbn:de:0111-pedocs-193299

https://doi.org/10.25656/01:19329

\section{Nutzungsbedingungen}

Gewährt wird ein nicht exklusives, nicht übertragbares, persönliches und beschränktes Recht auf Nutzung dieses Dokuments. Dieses Dokument ist ausschließlich für den persönlichen, nicht-kommerziellen Gebrauch bestimmt. Die Nutzung stellt keine Übertragung des Eigentumsrechts an diesem Dokument dar und gilt vorbehaltlich der folgenden Einschränkungen: Auf sämtlichen Kopien dieses Dokuments müssen alle Urheberrechtshinweise und sonstigen Hinweise auf gesetzlichen Schutz beibehalten werden. Sie dürfen dieses Dokument nicht in irgendeiner Weise abändern, noch dürfen Sie dieses Dokument für öffentliche oder kommerzielle Zwecke vervielfältigen, öffentlich ausstellen, aufführen, vertreiben oder anderweitig nutzen.

Mit der Verwendung dieses Dokuments erkennen Sie die Nutzungsbedingungen an.

\section{Terms of use}

We grant a non-exclusive, non-transferable, individual and limited right to using this document.

This document is solely intended for your personal, non-commercial use. Use of this document does not include any transfer of property rights and it is conditional to the following limitations: All of the copies of this documents must retain all copyright information and other information regarding legal protection. You are not allowed to alter this document in any way, to copy it for public or commercial purposes, to exhibit the document in public, to perform, distribute or otherwise use the document in public.

By using this particular document, you accept the above-stated conditions of use.

\section{Kontakt / Contact:}

peDOCS

DIPF | Leibniz-Institut für Bildungsforschung und Bildungsinformation Informationszentrum (IZ) Bildung

E-Mail: pedocs@dipf.de

Internet: www.pedocs.de 
Markus N. Sauerwein

\section{Qualitätsmerkmale in Ganztagsangeboten aus Perspektive der Schüler/innen}

\section{Zusammenfassung:}

In bisherigen empirischen Studien wurde die Qualität von Ganztagsangeboten über aus der Unterrichtsforschung adaptierte Qualitätsdimensionen erfasst. Durch diesen Ansatz werden allerdings die Potenziale außerschulischer Bildungsarrangements in der Ganztagsschule nicht genügend berücksichtigt. Daher sollten weitere, aus der Sozialpädagogik übernommene, Qualitätskriterien in Ganztagsangeboten mit einbezogen werden. Basierend auf einer Teilstudie der Studie zur Entwicklung von Ganztagsschulen (StEG-S) kann gezeigt werden, dass über Schülerfragebogen sechs voneinander zu trennende Qualitätsdimensionen in Ganztagsangeboten zu erfassen sind. Drei dieser Qualitätsdimensionen sind aus der Unterrichtsforschung (Classroom Management, kognitive Aktivierung und Autonomieunterstützung) und drei aus der Sozialpädagogik (Alltagsweltorientierung, Partizipation und Anerkennung) entnommen, wobei die Dimension Anerkennung nochmals in drei Teilaspekte unterteilt wird.

Schlüsselworte: Ganztagsangebote, Angebotsqualität, Ganztagsschule

\section{Perceived Aspects of Quality in extracurricular activities}

So far, empirical studies have assessed the quality of extracurricular activities based on dimensions of quality adapted from teaching research. This approach has been criticized because of a possible loss of the extracurricular potential contained in all-day opportunities. Rather, additional quality criteria should be considered for extracurricular activities, taken from social pedagogy work. Drawing on a part study from the Study on the Development of All-day Schools (StEG-S), it is possible to demonstrate that via student questionnaire, six separate quality dimensions can be assessed for extracurricular activities. Three of these dimensions are rooted in teaching quality research (classroom management, cognitive activation and autonomy support), the other three are taken from social pedagogy (lifeworld orientation, participation and recognition), whereas recognition can subdivide in three separate parts

Keywords: max. extracurricular activities, Quality in extracurricular activities, all-day-school; 


\section{Qualitätsmerkmale in Ganztagsangeboten - Unterrichtstheoretische und sozialpädagogische Zugänge}

Während der Gegenstand Unterricht in der Erziehungswissenschaft stark beforscht ist, scheint der Gegenstand Ganztagsangebot ein vergleichsweise unbekanntes Terrain zu sein. In der quantitativ-empirisch arbeitenden Unterrichtsforschung konnten über die vergangenen Dekaden, basierend auf den Arbeiten von Klieme und Kollegen (Klieme, Lipowsky, Rakoczy \& Ratzka 2006; Klieme \& Rakoczy 2008; Klieme, Pauli \& Reusser 2009; Helmke \& Klieme 2008; Klieme, Schümer \& Knoll 2001), drei sogenannte genuine Basisdimensionen guten Unterrichts erschlossen werden, die für motivationale und kognitive Prozesse im Unterricht bedeutsam sind: Das Classroom Management (bzw. eine effektive Zeitnutzung), ein motivationsunterstützendes schülerorientiertes Sozialklima (welches die Grundbedürfnisse nach sozialer Eingebundenheit, Kompetenz- und Autonomieerleben umfasst) sowie kognitiv aktivierende Frage- und Aufgabenstellungen.

In vorliegenden empirischen Arbeiten wurden diese drei Dimensionen guten Unterrichts auf Ganztagsangebote adaptiert (Fischer et al. 2011; Kielblock et al. 2014; Radisch 2009). Dies ist jedoch insofern problematisch, als sich dadurch Ganztagsangebote zu einem bestimmten Grad an den Standards von Unterricht bzw. Schule orientieren und somit die Gefahr besteht, Standards außerschulischer Bildung zu vernachlässigen. Insbesondere Vertreter aus der Sozialpädagogik respektive Jugendarbeit standen der breiten Einführung von Ganztagsschulen auch deshalb kritisch gegenüber, weil sie befürchteten, dass Ganztagsangebote eine Ausweitung von Schule bedeuten könnten und demnach die spezifischen Stärken und Ziele außerunterrichtlicher Bildungsarrangements verloren gehen würden (Otto \& Rauschenbach 2008; Rauschenbach 2009). In diesem Kontext wurde unter dem Schlagwort „Ganztagsbildung“ der Anspruch an Ganztagsschulen formuliert, mehr zu sein als eine Ausweitung des Unterrichts oder unterrichtsähnlicher Settings über den ganzen Tag und stattdessen andere Bildungsmöglichkeiten zu berücksichtigen. Gefordert wurde, dass die Schulen mit außerschulischen Bildungsakteuren zusammenarbeiten sollten, insbesondere der Jugendarbeit, sodass in wechselseitiger Ergänzung die Stärken beider zum Tragen kommen (Coelen 2002; 2006; Rauschenbach 2003).

Als ihre genuine Stärke versteht Jugendarbeit den freiwilligen und offenen Charakter ihrer Bildungsangebote. Sie konzipiert sich selbst als Feld der politischen und kulturellen Bildung. Lernen findet nicht formell statt, sondern durch die informelle Auseinandersetzung mit der eigenen Alltagswelt, der Gesellschaft, der Peergruppe, den Betreuer/innen etc. Ziel der Jugendarbeit ist, dass sich Jugendliche über Aushandlungsprozesse und Partizipationsmöglichkeiten zu kritischen und mündigen Staatsbürgern entwickeln, die fähig sind, sich aktiv in die Gesellschaft einzubringen und ihre eigenen Meinungen reflektieren und revidieren können. Kennzeichnend für die Jugendarbeit ist ein (emanzipatorischer) Bildungsbegriff, der die Selbsttätigkeit, Freiwilligkeit und Eigenverantwortung betont (Sturzenhecker 2004; Kentler 2006; Thole 2000). Bildung in der Jugendarbeit meint immer den Prozess des „Sich-Bildens“ und ist deshalb immer Selbstbildung. Bildung ist mehr als eine Zusammenstellung von Wissen und geht über den reinen Qualifikationserwerb hinaus. Als Lebenskompetenz bzw. als Befähigung zur eigenbestimmten Lebensführung (Bundesjugendkuratorium 2002) könnte die Doktrin der Kinder- und Jugendarbeit pointiert werden. Folglich stehen Prozesse der Anerkennung, Selbstentfaltung und Selbstbestimmung im Mittelpunkt (Rauschenbach et al. 2010, 237; Thole 2013). Werte, Normen und soziale Kompetenzen werden als direkt erfahrbare und reflektierbare Inhalte der Jugendarbeit verstanden, die keinen zu erlernenden Wissenskanon darstellen (Schröder 2005), sondern 
unter den Realbedingungen des Alltags angeeignet werden können (Rauschenbach 2009, 196). Übergeordnete Ziele der Kinder- und Jugendarbeit, wie Selbstbestimmung und Verantwortungsübername, sollen direkt durch die Möglichkeiten der Partizipation erlernt werden (Zinser 2005; Müller, Schmidt \& Schulz 2005, 16f.). Dadurch sollen Heranwachsende, fernab von Zwang, dazu angeregt werden, alle Potenziale auszubilden, um eine eigene Persönlichkeit entfalten zu können.

Gut 15 Jahre nach dem im Rahmen des Investitionsprogramms Zukunft, Bildung und Betreuung (IZZB) geförderten Ganztagsschulausbaus in Deutschland zeigt sich, dass es der Jugendarbeit gelungen ist, in Ganztagsschulen als Kooperationspartner mitzuwirken (Bundesbericht 2012/2015). Jedoch scheint sie sich hierfür, zumindest in Teilen, schulischen Rahmenbedingungen und (Bildungs-)Zielen unterzuordnen. So versuchen beispielsweise Angebote der Jugendhilfe im Kontext von Ganztagsschule den Schulerfolg mit abzusichern, was nicht ihrem ursprünglichen Bildungsauftrag entspricht (Zipperle 2015). Ferner scheint es der Jugendarbeit nicht gelungen zu sein, ihr eigenes Qualitätsverständnis in die Ganztagsschule zu transportieren. Hinzu kommen Positionen, nach denen „ganztägige Schulen [...] zunächst einen gesellschaftlichen Beitrag zur Sicherstellung und zur Qualitätssteigerung der soziokulturellen Infrastruktur (z. B. Jugendarbeit) dar [stellen]" (Höhmann et al. 2005, 170). Es überrascht kaum, dass solche Aussagen aus Perspektive der Jugendarbeit kritisch gesehen werden, wird hierüber lanciert, dass Ganztagsschule eine Qualitätssteigerung für (ehemals) außerschulische Angebote der Jugendarbeit bedeutet (Coelen \& Wahner-Liesecke 2009 , 810). Vor diesem Hintergrund scheint die Befürchtung nachvollziehbar, dass außerschulische Bildungsarrangements ihre genuinen Stärken und Vorteile gegenüber dem Schulunterricht, durch die breite Einführung von Ganztagsschulen, verlieren können.

Gleichzeitig ist die pädagogische Gattung Ganztagsangebot als solche bisher wenig erforscht und es ist unklar, wo diese im Feld zwischen Unterricht und außerschulischen Lernorten zu verorten ist. Einigkeit scheint darüber zu bestehen, dass es sich hierbei weder um Unterricht noch um gänzlich außerschulische Bildungsangebote handelt. Anders formuliert: Ganztagsangebote sind etwas Neues - weder Unterricht noch sozialpädagogisches Angebot oder außerschulische Freizeitbeschäftigung. Dementsprechend erscheint es weniger eindeutig, was Qualitätsmerkmale von Ganztagsangeboten sind bzw. sein könnten. Ein alleiniger Fokus auf die Qualitätsdimensionen guten Unterrichts würde die Bildungspotenziale von Ganztagsangeboten nur unzureichend abdecken

Der Idee der Ganztagsbildung folgend, könnte ein Ansatz zur Beschreibung der Qualität von Ganztagsangeboten darin bestehen, sowohl aus der Unterrichtsforschung als auch aus der Sozialpädagogik Qualitätsmerkmale für Ganztagsangebote zu adaptieren. Neben den Basisdimensionen guten Unterrichts könnten aus der Sozialpädagogik Altagsweltorientierung, Partizipation sowie unterschiedliche Formen von Anerkennung als Merkmale von Ganztagsangeboten herangezogen werden. Diese werden in der sozialpädagogischen Fachliteratur nicht explizit als "Qualitätsdimensionen" ausgeführt, sind jedoch Aspekte die wiederholt benannt werden und wurden deshalb im Rahmen der Studie zur Entwicklung von Ganztagsschulen (StEG) als Qualitätsdimensionen für Ganztagsangebote aufgegriffen.

Zusammenfassend erscheint es demnach vollständiger und angemessener, sowohl die Basisdimensionen guten Unterrichts als auch Qualitätsmerkmale der Sozialpädagogik zu berücksichtigen, um Qualitätsmerkmale von Ganztagsangeboten erfassen zu können, die nachfolgend kurz beschrieben werden. Die ersten drei Merkmale sind der Unterrichtsforschung entnommen, die letzten drei Merkmale der Sozialpädagogik, wobei die 
Qualitätsdimension Anerkennung in drei Subfacetten aufgeteilt wird. Insbesondere beim Aspekt der Schüler/innen-Orientierung darf diskutiert werden, inwiefern es hier zu Überschneidungen mit den sozialpädagogischen Qualitätsdimensionen kommt (s. hierzu Inhaltliche Überschneidungen):

- Unter Classroom Management wird eine strukturierte und störungspräventive Unterrichtsführung verstanden, die darauf abzielt, die vorhandene Unterrichtszeit möglichst optimal zum Unterrichten und Lernen zu verwenden. Deshalb wird hierbei auch von einer effektiven Zeitnutzung gesprochen (Kounin 2006; Klieme \& Rakoczy 2008, Seidel 2009; Kunter \& Trautwein 2013).

- Die Motivationsunterstützung bzw. Schüler/innen-Orientierung berücksichtigt die natürlichen Bedürfnisse der Schüler/innen nach Sicherheit, Autonomie und Kompetenzerleben (Deci \& Ryan 1993). Lehrkräfte sollten Schüler/innen das Gefühl von Autonomie geben, sie emotional und sozial unterstützen sowie einen alltagsweltlichen Bezug zu den Unterrichtsthemen herstellen. Im Unterricht besteht hier die Herausforderung, diese Aspekte trotz vorstrukturierter Lehrpläne umzusetzen (Rakoczy 2006; Kunter 2005)

- Kognitive Aktivierung bezieht sich auf herausfordernde und anregende Aufgabenbzw. Fragestellungen, die Schüler/innen dazu anregen sollen, sich kognitiv mit der Materie des Unterrichts zu beschäftigen, um dabei tiefergehende Lernprozesse anzustoßen (Klieme \& Rakoczy 2008; Mayer 2002). Die grundlegende lerntheoretische Prämisse des Konstruktes kognitive Aktivierung darf dabei wie folgt pointiert werden: „Je stärker Lernende sich mental aktiv mit dem Lerngegenstand auseinandersetzen, umso besser werden Konzepte verstanden und umso nachhaltiger wird das Lernen" (Kunter \& Trautwein 2013, 86).

- Alltagsweltorientierung meint eine Orientierung an den Cliquen, Szenen und Ritualen sowie den Wünschen, Bedürfnissen und Fähigkeiten von Kindern und Jugendlichen (u.a. Sting \& Sturzenhecker 2005; Thole 2000; achter Kinder- und Jugendbericht 1990). Dies bedeutet, die Kinder ernst zu nehmen und ihre Alltagsdeutungen zu akzeptieren sowie regionale und milieuspezifische Besonderheiten zu bedenken (Thole 2000, 260). Bereits im achten Jugendbericht (1990) wurde gefordert, falls Schulen Angebote für den Freizeitbereich entwickeln, sich diese an den Lebensbedingungen der Schüler/innen orientieren müssen.

- Um übergeordnete pädagogische Ziele wie Selbstbestimmung, Verantwortungsübername und demokratische Mitbestimmung zu erreichen, benötigen Kinder und Jugendliche entsprechende Gelegenheitsstrukturen (Sting \& Sturzenhecker 2005). Partizipation ist sicherlich eine dieser Möglichkeiten, die am häufigsten benannt wird (Zinser, 2005). In pädagogischen Settings müssen deshalb ausreichend Partizipationsmöglichkeiten für die Kinder und Jugendlichen bereitgestellt werden, damit Partizipation erprobt und erlernt werden kann (Zinser, 2005; Sting \& Sturzenhecker, 2005).

- Anerkennung gilt als Voraussetzung für die Identitätsbildung und -findung, Subjektbildung, Selbstentfaltung und den Aufbau von Selbstwertgefühl (u.a. Balzer \& Ricken 2010; Schoneville \& Thole 2009; Ricken 2006; Scherr 2002). Die Befähigung zur Selbstachtung und gegenseitigen Anerkennung wird immer wieder als Zielsetzung der Pädagogik aufgeführt (Prengel 2006; Scherr 2002). Dabei wird Anerkennung in drei Dimensionen differenziert: Emotionale Zuwendung (Liebe), Individuelle Anerkennung (Solidarität) und Recht (bzw. Missachtung). 
- Bei emotionaler Zuwendung geht es um die grundlegende Anerkennung der Einzelperson und der Achtung der Persönlichkeit dieser (Prengel 2006). Eine positive, interessierte, freundliche und offene Haltung gegenüber Jugendlichen sollte in einem professionelle pädagogischen Alltag grundlegend sein (Helsper \& Lingkost 2002, S. 132f.; Helsper et al. 2001). Jugendlichen mit problematischen Einstellungen oder Verhaltensweisen sollte nicht stigmatisierend oder ausgrenzend gegenübergetreten werden, sondern mit emotionaler Anerkennung (Thole \& Schoneville 2010; Schoneville \& Thole 2009).

- In der Sphäre individueller Anerkennung sollen die spezifischen Eigenschaften und Fähigkeiten des einzelnen Subjekts betrachtet und wertgeschätzt werden (Reinhardt 2002; Honneth 1994, 210; Helsper et al. 2001, 33). Für Adoleszenten bedeutet dies, dass sie Anerkennung für ihre Leistungen, Eigenschaften, Lebensstilen und Selbstdarstellungsformen erhalten, die sie von anderen unterschiedlich (Bettmer 2009; Helsper \& Lingkost 2002, 136).

- Im pädagogischen Kontext müssten rechtliche Anerkennungsformen (bzw. Missachtung) als eine „Form von gerechter Behandlung und Zubilligung gleicher Rechte“ (Helsper \& Lingkost 2002, 133; Helsper et al. 2001, 32) verstanden werden. Die Normalitätskonzeption in pädagogischen Settings besteht darin, dass die Pädagogen bestimmte (rechtliche - nicht zwangsweise auch juristische) Standards einhalten, d.h. sie versuchen, die Heranwachsenden gleich und fair zu behandeln. Dazu gehört, dass sie die Schüler/innen nicht bloßstellen, anschreien und sie körperlich nicht angreifen. Missachtungen dieser Standards können als rechtswidrige Übergriffe aufgefasst werden.

\section{Inhaltliche Überschneidung}

Auf theoretischer Ebene ist ferner zu diskutieren, in welchem Verhältnis das Qualitätsmerkmal Schüler/innen-Orientierung bzw. Motivationsunterstützung (Unterrichtsforschung) zu den Dimensionen Partizipation, Alltagsweltorientierung und Anerkennung steht. Die Dimension der Schüler/innen-Orientierung beinhaltet Aspekte von sozialer Eingebundenheit, Autonomie und Kompetenzerleben. Konkret muss das Verhältnis zwischen Autonomieunterstützung zu Partizipation und Alltagssweltorientierung (1), zwischen sozialer Eingebundenheit und den Anerkennungsdimensionen Emotionale Zuwendung (Liebe) und Recht (2) sowie zwischen Kompetenzunterstützung und der Anerkennungsform Soziale Wertschätzung (3) skizziert werden:

(1) Autonomieunterstützung im Unterricht basiert auf der Annahme, dass Freiräume, Wahlmöglichkeiten, Partizipationsgelegenheiten und eine persönliche Bedeutung einer Aufgabe das Autonomieerleben der Schüler/innen positiv beeinflussen können. Vor dem Hintergrund des Angebots-Nutzungs-Modells können Partizipationsgelegenheiten als Angebot an die Schüler/innen verstanden werden, welches von ihnen genutzt werden kann und sich im optimalen Fall im Autonomieerleben äußert. Beides, Autonomieunterstützung und Partizipationsmöglichkeiten können demnach das Autonomieerleben positiv beeinflussen. Der Unterschied zwischen beiden Dimensionen besteht darin, dass 
Autonomieunterstützung den einzelnen Schüler/ die einzelne Schülerin adressiert, während Partizipation nur im Kontext einer Gruppe möglich ist.

Partizipationsgelegenheiten sollen demnach auch auf ein Leben in der Demokratie vorbereiten, während Autonomieunterstützung vornehmlich das Autonomieerleben und damit intrinsische Motivation bei Schüler/innen hervorrufen soll, wodurch letztlich der Lernerfolg gesteigert werden kann.

Bei dem Konstrukt der Alltagsweltorientierung ist auf einen direktionalen Unterschied zur Autonomieunterstützung hinzuweisen: Assor et al. (2002) schreiben, dass Lehrer/innen autonomieunterstützend handeln, wenn sie Schüler/innen die persönliche Relevanz einer Aufgabenstellung darlegen können. Dies bedeutet, dass die Aufgabe eine Alltagsweltliche Aufladung erhalten soll. Den Schüler/innen wird verdeutlicht, weshalb diese Aufgabe für ihr Leben relevant ist. Das Konzept der Alltagsweltorientierung meint dies jedoch nicht. Die Ausgangslage ist genau umgekehrt: Jugendliche werden im Kontext ihrer Alltagswelt betrachtet und es wird gefragt, wie sie diese bewältigen können, d.h. Jugendliche können Alltagserfahrungen ihrer Alltagswelt einbringen, sodass diese thematisiert werden. D.h. es bestehen keine vorgefertigten Aufgaben, deren Bedeutung Jugendlichen erklärt werden sollen, sondern die Jugendlichen können „die Aufgaben“ selbst einbringen.

(2) Soziale Eingebundenheit und emotionale und rechtliche Anerkennung beschreiben letztlich ein ähnliches Verständnis von Pädagogik. Auf diese Überschneidung weist auch Prengel (2013) hin. Pädagogen sollten Kindern und Jugendlichen respektvoll und empathisch gegenübertreten. Dies kann als grundlegend für erfolgreiches Lernen und für eine gesunde Persönlichkeitsentwicklung betrachtet werden. Deshalb wird in der Anerkennungstheorie darauf insistiert, dass emotionale Anerkennung bedingungslos erfolgen muss. Ferner bietet die in der Anerkennungstheorie aufgezeigte Differenzierung zwischen emotionaler und rechtlicher Anerkennung einen analytischen Mehrwert gegenüber dem Konstrukt der sozialen Eingebundenheit, weshalb es als sinnvoll erscheint, für Ganztagsangebote auf das Anerkennungskonzept zu rekurrieren.

(3) Bei der Kontrastierung zwischen Kompetenzunterstützung und individueller Anerkennung könnte die eben aufgestellte Diskussion wiederholt werden: Kompetenzunterstützung fokussiert stärker auf den Leistungsaspekt, während soziale Wertschätzung auf die Möglichkeit rekurriert, dass Individuen sich positiv auf ihre eigenen Fähigkeiten (kognitive und non-kognitive) beziehen können, um sich als eine von anderen unterscheidbare Person zu begreifen und hierüber zu erfahren, als Person einen Wert für die Gesellschaft zu erhalten. Die Reichweite der anerkennungstheoretischen Konzeption scheint hierbei eine größere Rolle zu spielen: Im pädagogischen Rahmen insistiert sowohl Kompetenzunterstützung als auch soziale Wertschätzung auf Formen individueller Förderung. Es geht darum, dass den Schüler/innen Aufgaben gestellt werden, die ihren individuellen Fähigkeiten entsprechen und für die Leistungsbeurteilung individuelle Bezugsnormen herangezogen werden. Kompetenzunterstützung ist hier jedoch in erster Linie auf kognitive Kompetenzen ausgerichtet, während individuelle Anerkennung vornehmlich eine positive Entwicklung des Subjekts einschließt bzw. konkreter, des Selbstwerts oder der Selbstwirksamkeit. 
Aus diesen drei Überlegungen heraus kann begründet werden, sowohl

Autonomieunterstützung als auch Alltagsweltorientierung, Partizipationsmöglichkeiten und die drei Formen von Anerkennung als einzelne Qualitätsdimensionen zu berücksichtigen. Alltagsweltorientierung ist nicht unter das Konzept der Autonomieunterstützung zu subsumieren, ebenfalls ist Partizipation ein eigenständiges Qualitätskonstrukt. Die drei Formen von Anerkennung beziehen sich nicht nur auf motivationale Variablen oder schulisch zu erlernende Kompetenzen und scheinen deshalb für Ganztagsangebote, die umfassendere Bildungsziele verfolgen, besser geeignet zu sein als Motivationsunterstützung (oder Kompetenzunterstützung).

\section{Fragestellung}

Der Zugang, die Qualität von Ganztagsangeboten über die aufgeführten Qualitätsmerkmale zu erfassen, ist bis zu dieser Stelle rein theoretischer Natur. Offen ist, ob diese Qualitätsmerkmale tatsächlich in unterschiedlichen Ganztagsangeboten auftreten und voneinander zu unterscheiden sind.

In diesem Beitrag werden deshalb die in der Studie zur Entwicklung von Ganztagsschulen (StEG) verwendeten Qualitätsskalen vorgestellt. Hierbei interessiert, ob die Qualitätsdimensionen aus der Unterrichtsforschung und Jugendarbeit in Ganztagsangeboten über Schüler/innen-Urteile erhoben und empirisch voneinander unterschieden werden können.

1. Zunächst wird mittels konfirmatorischer Faktorenanalyse betrachtet, ob acht differenzierte Qualitätsdimensionen unterschieden werden können oder die Angebotsqualität global bewertet wird.

2. Zweitens werden Korrelationen zwischen den Angebotsqualitätsdimensionen berechnet, um zu eruieren, inwiefern es zwischen einzelnen Qualitätsdimensionen zu Überschneidungen kommt.

Die Analysen werden exemplarisch für drei Arten von Ganztagsangeboten durchgeführt: Erstens für Leseangebote, in denen Schüler/innen zielgerichtet ihre Lesekompetenzen verbessern sollen. Zweitens für Medienangebote, wie Theater-Gruppe, Schulzeitung/Homepage etc., in denen Lesefähigkeiten und soziale Kompetenzen informell angeeignet werden können. Drittens für Angebote zum sozialen Lernen, wie beispielsweise Streitschlichter, LionsQuest, Coolness Training etc., in denen soziale Kompetenzen und der Umgang mit Konfliktsituationen erlernt werden sollen.

Abschließend wird diskutiert, inwiefern die hier vorgestellten Skalen zur Erfassung der Qualität in Ganztagsangeboten auch von Praktikern eingesetzt werden können und worauf hierbei zu achten ist.

\section{Studiendesign, Stichprobe und Methode}

Die zuvor aufgeworfenen Fragen sollen mit Hilfe der Studie zur Entwicklung von Ganztagsschulen (StEG) beantwortet werden. StEG ist ein Kooperationsprojekt zur Analyse der Entwicklung der deutschen Ganztagsschullandschaft, welches vom Bundesministerium für Bildung und Forschung (BMBF) finanziert wird. Nach der ersten Förderphase (20052011) wurde das Projekt bis 2015 verlängert. Die empirischen Daten der vorliegenden Arbeit stammen aus der Studie zur Entwicklung von Ganztagsschulen (StEG-S), die ein Teilprojekt von StEG ist und am Deutschen Institut für Internationale Pädagogische Forschung (DIPF) konzipiert und durchgeführt wurde. In StEG-S wurden Schüler/innen im Verlauf ihres ersten Schuljahres in der Sekundarstufe (5. Klasse) drei Mal mithilfe von Fragebogen am 
Computer befragt: zu Schuljahresbeginn, zum Schulhalbjahr sowie am Ende der fünften Klasse. Zum zweiten und dritten Messzeitpunkt wurden die Schüler/innen zur Qualität des Unterrichts im vergangen Halbjahr sowie, im Falle des Besuchs von Ganztagsangeboten, zur Qualität dieser befragt.

Insgesamt konnten 66 Schulen für die Teilnahme an StEG-S gewonnen werden. Im Vorfeld des zweiten und dritten Messzeitpunktes wurden diese gebeten, mitzuteilen, welche Ganztagsangebote an ihrer Schule für die fünfte Klasse durchgeführt werden. Basierend auf diesen Angebotslisten wurden die Ganztagsangebote zu Kategorien zusammengefasst. Den Schüler/innen selbst wurden zur Beurteilung der Qualität der Angebote jedoch nicht die abstrakten Kategorien vorgelegt, sondern die konkreten Namen der Angebote, wie sie an ihrer Schule geläufig waren. Die Schüler/innen wurden also nicht gefragt, ob sie ein Medienangebot besuchen, sondern ob sie z. B. bei der „Online Schulzeitung“ mitwirken. Gehörte eines der ausgewählten Ganztagsangebote zu den forschungsrelevanten Kategorien (Medien, Lesen, soziales Lernen), wurden die Schüler/innen anschließend gebeten, die Qualität des Ganztagsangebots zu beurteilen. Für den Fall, dass bestimmte Schüler/innen mehrere forschungsrelevante Ganztagsangebote besuchten, gab es eine entsprechende Priorisierung, weil aus zeitlichen Gründen nicht die Qualität aller besuchten Ganztagsangebote bewerten konnte.

\subsection{Stichprobe}

Über alle drei Messzeitpunkte hinweg konnten an den 66 Schulen insgesamt 2105 Schüler/innen mittels Fragebogen befragt werden. Zum zweiten und dritten Messzeitpunkt fiel die Anzahl der befragten Schüler/innen etwas geringer aus (2.MZP: 1969 | 3.MZP: 1959). Insgesamt verteilen sich die Schüler/innen auf die Schulformen Hauptschule (5,6\%), Realschule (6,8\%), Schule mit mehreren Bildungsgängen (30,8\%), Gesamtschule $(26,6 \%)$ sowie Gymnasium (30,3\%). 54 Prozent der befragten Schüler/innen waren männlich. Laut Angaben der Schulleiter/innen war die Teilnahme am Ganztagsbetrieb an 20 der 66 Schulen für alle Schüler/innen verbindlich. Damit besuchten knapp ein Drittel der befragten Schüler/innen (29,9\%) eine „gebundene“ Ganztagsschule. Die Anzahl der Schüler/innen, die für die Studie relevante Ganztagsangebote besuchen bzw. bewerten, ist allerdings als eher gering einzuschätzen. Je nach Angebotsart und Messzeitpunkt schwankt die Zahl der Schüler/innen zwischen 72 (Leseangebote 3. MZP) und 142 (Medienangebote 2. MZP). Je Angebot nehmen demnach nur etwa 5 Prozent der Schüler/innen in den für die Studie relevanten Ganztagsangeboten teil (vgl. Tabelle 1). Die Analysen für die Beurteilung der Angebotsqualität beruhen demzufolge auf einer kleinen Stichprobe.

Tab. Nummer (1): Schüler/innen in Medienangebote, Leseangeboten bzw. Angeboten zum sozialen Lernen

\begin{tabular}{|l|l|l|l|}
\hline $\begin{array}{l}\text { Anzahl Schüler/innen } \\
\text { in Angeboten }\end{array}$ & Medienangebote & Leseangebote & $\begin{array}{l}\text { Angebote zum } \\
\text { sozialen Lernen }\end{array}$ \\
\hline 2. MZP & $142(6,7 \%)$ & $118(5,6 \%)$ & $104(4,9 \%)$ \\
\hline $3 . \mathrm{MZP}$ & $136(6,5 \%)$ & $72(3,6 \%)$ & $123(5,8 \%)$ \\
\hline
\end{tabular}

\subsection{Instrumente zur Erfassung der Angebotsqualität}

Um das heuristische Konzept Ganztagsangebote als eine pädagogische Gattung zwischen Unterricht und außerschulischen Bildungsarrangements ansehen zu können, wurden Qualitätsdimensionen für Ganztagsangebote operationalisiert, d.h. die Facetten der 
jeweiligen Qualitätsdimensionen wurden über verschiedene Fragen (Items) an die Schüler/innen abgebildet. Fragen, die ein Qualitätsmerkmal abbilden, werden dabei zu einer Skala zusammengefasst. Schüler/innen wurden gefragt "Wie ist das bei dir im Angebot? Was meinst du? Ich finde im Angebot [Name eines zuvor gewählten Angebots]..."1

Tab. Nummer (2): Operationalisierung der Qualitätsdimensionen für Ganztagsangebote

Zeitnutzung (Quelle: Angelehnt an DESI 2003 - Wagner, Helmke, Rösner)

a) ...wird fast immer konzentriert gearbeitet.

b) ...wird häufig Zeit verschwendet.

c) ...dauert es lange, bis alle Schülerinnen und Schüler arbeiten.

d) ...kommen wir immer sofort zur Sache.

Kognitive Aktivierung (Quelle: Angelehnt an DESI 2003 - Wagner, Helmke, Rösner)

d) ...möchte, dass wir unsere Antworten auch erklären können.

e) ...kann Schülerinnen und Schüler manchmal richtig begeistern.

f) ...stellt Fragen, über die ich nachdenken muss.

Autonomie Erleben (Quelle: Angelehnt an PISA 2003)

a) ...habe ich die Möglichkeit, ein Thema eigenständig zu bearbeiten.

b) ...ist es mir möglich, meine Zeit selbst einzuteilen.

c) ...habe ich das Gefühl, dass ich eigene Entscheidungen treffen kann.

Partizipation (Quelle: Angelehnt an DESI 2003 - Wagner, Helmke, Rösner)

a) ...geht auf unsere Vorschläge ein.

b) ...ermutigt uns, unsere eigene Meinung auszudrücken.

c) ...geht darauf ein, wenn jemand eine gute Idee hat.

Alltagsweltorientierung (Quelle: Angelehnt an MADICS 1990 - 2000)

d) ...können wir eigene Erfahrungen und Beispiele aus dem täglichen Leben einbringen.

e) ...wird auf vieles aus meinem täglichen Leben eingegangen.

f) ...lerne ich Dinge, die mir im täglichen Leben helfen.

Anerkennung: Emotional (Quelle: Eigene Entwicklung)

a) ... nimmt mich ernst.

\footnotetext{
${ }^{1}$ Als auf den/die Betreuer/in bezogener Prompt wurde folgende Eingangsfrage verwendet: Denke nun an die erwachsene Person, die das Angebot [Name eines zuvor gewählten Angebots] leitet, wir nennen diese Person Betreuer/in. Ich finde, meine Betreuerin/ mein Betreuer...".
} 
b) ... akzeptiert mich so wie ich bin.

c) ... kann ich vertrauen.

d) ... Betreuer komme ich gut aus.

Anerkennung: Individuell (Quelle: Eigene Entwicklung)

a) ...kümmert sich um mich.

b) ...erkennt meine Stärken.

c) ...schätzt meine Fähigkeiten.

d) ...bietet mir die Möglichkeit zu zeigen, was ich kann.

Anerkennung: Recht / Missachtung (Quelle: Eigene Entwicklung)

e) ...hat sich schon mal vor anderen Kindern über mich lustig gemacht.

f) ...hat mich schon mal angeschrien.

g) ....behandelt mich ungerecht.

\subsection{Methoden}

Quantitative Forschungsmethoden versuchen über einzelne Fragen (Items) ein latentes, d.h. nicht direkt beobachtbares, Konstrukt zu erheben. So wird beispielsweise davon ausgegangen, dass die kognitive Aktivierung in Ganztagsangeboten nicht direkt zu beobachten ist, sondern sich aus verschiedenen Indikatoren (Items) zusammensetzt, die Teilaspekte des latenten Faktors erklären. Jedes Item ist hierbei für den entsprechenden Faktor von unterschiedlicher Relevanz - dies wird, methodisch gesprochen, über die Faktorladung angezeigt. Für die unterschiedlichen Qualitätskonzepte wurden in StEG-S verschiedene Items konzipiert (vgl. Tab. 2). Diese sollten nun zur Erklärung des jeweils ihnen zugehörigen latenten Konstruktes beitragen. Denkbar wäre auch, dass die theoretisch aufgemachte Trennung der verschiedenen Qualitätskonzepte empirisch nicht haltbar ist und Schüler/innen die Angebotsqualität eher global bewerten, d.h. alles in einem Angebot als gut oder schlecht bewerten und nicht zwischen den einzelnen Qualitätsaspekten differenzieren. In diesem Fall könnten die Items nicht ihrem jeweiligen Qualitätskonzept zugeordnet werden, sondern nur einem globalen undifferenzierten Qualitätsfaktor. Um dies zu prüfen, werden sogenannte konfirmatorische Faktorenanalysen (CFA) durchgeführt, indem ein Modell, welches einen globalen Qualitätsfaktor annimmt, gegen ein Modell getestet wird, das zwischen verschiedenen Qualitätsdimensionen unterscheidet. Alle Analysen wurden In MPlus 7 \{Muthén 1998 - 2012 \#1014\} durchgeführt, wobei der WLSMV-Schätzer verwendet wurde, um das kategoriale Skalenniveau zu berücksichtigen. ${ }^{2}$

Zusätzlich werden Korrelationen zwischen den Qualitätsdimensionen berechnet.

Korrelationen zeigen an, wie die unterschiedlichen Qualitätsdimensionen zusammenhängen. Zudem können hohe Korrelationen ( $r>.9$ ) der einzelnen Qualitätsskalen untereinander als

\footnotetext{
2 Der Weighted Least-Squares Estimation of Mean- and Variance (WLSMV) Schätzer wurde verwendet, weil dieser auch bei kleineren Stichproben gut funktioniert (Sass 2011), gleichzeitig bei einem vierstufigen Antwortniveau nicht von einer Metrik ausgegangen werden darf (Desa 2014).
} 
Indiz dafür angesehen werden, dass eine Differenzierung zwischen zwei Qualitätsdimensionen empirisch nicht haltbar ist.

\section{Ergebnisse}

Um zu überprüfen, ob die Schüler/innen die Qualität in Unterricht und Ganztagsangeboten eher global wahrnehmen oder basierend auf ihren Antworten, zwischen den theoretisch angenommenen Qualitätsmerkmalen differenziert werden kann, wurde geprüft, ob ein eindimensionales oder ein achtdimensionales Modell die empirischen Daten besser widerspiegelt. Entsprechend wurde ein Modell, in dem alle Items auf einen Faktor laden, verglichen mit einem Modell, in dem die Items den acht theoretisch angenommenen Qualitätsdimensionen zugeordnet wurden. Negativ formulierte Items wurden hierfür recodiert und ein Methodenfaktor mit in die Analysen aufgenommen, der das Antwortverhalten der Schüler/innen berücksichtigt. Die Ergebnisse der konfirmatorischen Faktorenanalysen bestätigen insgesamt, dass sich die Qualitätsdimensionen in den drei verschiedenen Angebotskategorien gut voneinander trennen lassen. Ein Modell, das eine achtdimensionale Faktorstruktur annimmt, ist einem eindimensionalen Modell überlegen, dafür sprechen die besseren CFI und RMSEA Werte ${ }^{3}$ sowie der signifikante Chi $^{2}$-Test ${ }^{4}$, der prüft, ob ein Modell mit einem Faktor signifikant schlechter auf die Daten passt als ein Modell mit acht eigenständigen Faktoren (Tabelle 3).

Die theoretisch aufgemachte Erweiterung und Unterscheidung in acht verschiedene Qualitätsdimensionen kann demnach empirisch gestützt werden. Dies gilt für alle drei betrachteten Angebotskategorien - Medien, Lesen und soziales Lernen.

\footnotetext{
${ }^{3}$ Ohne im Detail auf die Bedeutung der sogenannten FIT-Werte einzugehen, gilt, dass ein CFI von $>.90$ als ausreichend und $>.95$ als gut bewertet wird. Bei dem RMSEA sind Werte $<.08$ als ausreichend und $<.05$ als gut zu interpretieren. Allerdings gibt es für den WLSMV-Schätzer noch keine Simulationsstudien, auf die sich bezogen werden kann und es wird u.a. diskutiert, dass der RMSEA bei diesem Schätzer nicht die gleiche Güte aufweist wie bei dem ML-Schätzer (Sass, Schmitt \& Marsh 2014; Desa, 2014).

${ }^{4}$ Der "normale" Chi ${ }^{2}$ Test kann für den WLSMV-Schätzer nicht verwendet werden aber in Mplus kann ein Chi Differenzen Test für den WLSMV-Schätzer (über die Option DIFFTEST) angefordert werden (Muthén 1998 2012; Sass 2011; Muthén \& Asparouhov 2002).
} 
Tab. Nummer (3): Acht getrennte Qualitätsdimensionen vs. einen eindimensionalen Qualitätsfaktor; Analysen mit WLSMV-Schätzer gerechnet

\begin{tabular}{|c|c|c|c|c|c|c|}
\hline Alle Qualitätsskalen & \multicolumn{2}{|c|}{ Medien } & \multicolumn{2}{|c|}{ Lesen } & \multicolumn{2}{|c|}{ Soziales Lernen } \\
\hline 2.MZP & 1 Faktor & 8 Faktoren & 1 Faktor & 8 Faktoren & 1 Faktor & 8 Faktoren \\
\hline CFI & 0.82 & .97 & 0.82 & .94 & 0.76 & .97 \\
\hline RMSEA & 0.11 & .04 & 0.10 & .062 & 0.14 & .06 \\
\hline \begin{tabular}{|l|} 
Chi $^{2}$ Test \\
1 Faktor vs. 8 Faktoren \\
\end{tabular} & \multicolumn{2}{|c|}{$313.404 * * *$} & \multicolumn{2}{|c|}{$244.472 * * *$} & \multicolumn{2}{|c|}{$339.954 * * *$} \\
\hline $\mathrm{N}$ & \multicolumn{2}{|c|}{141} & \multicolumn{2}{|c|}{114} & \multicolumn{2}{|c|}{97} \\
\hline 3.MZP & 1 Factor & 8 Faktoren & 1 Factor & 8 Faktoren & 1 Factor & 8 Faktoren \\
\hline CFI & .90 & .98 & 0.89 & .95 & 0.78 & .93 \\
\hline RMSEA & .11 & .06 & .12 & .09 & 0.14 & .08 \\
\hline $\begin{array}{l}\mathrm{Chi}^{2} \text { Test } \\
1 \text { Faktor vs. } 8 \text { Faktoren } \\
\end{array}$ & \multicolumn{2}{|c|}{$284.005 * * *$} & \multicolumn{2}{|c|}{$160.218 * * *$} & \multicolumn{2}{|c|}{$328.166^{* * *}$} \\
\hline $\mathrm{N}$ & \multicolumn{2}{|c|}{131} & \multicolumn{2}{|c|}{76} & \multicolumn{2}{|c|}{116} \\
\hline
\end{tabular}

$\mathrm{CFI}=$ Comparative Fit Index); RMSEA = Root Mean-Square Error of Approximation ${ }^{* * *} p<.001$

Zusätzlich zu den konfirmatorischen Faktorenanalysen wurden Korrelationen zwischen den einzelnen Qualitätsdimensionen für die Ganztagsangebote zu den jeweiligen Messzeitpunkten betrachtet. Die Ergebnisse können ebenfalls Aufschluss darüber geben, inwiefern eine Trennung der Qualitätsdimensionen sinnvoll ist. Im Prinzip bestätigen die Korrelationen die Ergebnisse der konfirmatorischen Faktorenanalysen: Zwar bestehen zwischen einzelnen Dimensionen starke Zusammenhänge ( $r>.5$ ) (vgl. Tabelle 4), jedoch sind keine Korrelationen zwischen den einzelnen Dimensionen von $r>.8$ zu erkennen. Folglich sind die einzelnen Qualitätsdimensionen untereinander nicht so hoch korreliert, dass darüber diskutiert werden könnte, sie in einer Skala zusammenzufassen.

Nachfolgend werden starke Zusammenhänge ( $r>.5)$ referiert, die zwischen einzelnen Qualitätsdimensionen in mehreren Ganztagsangeboten auftreten. Beispielsweise fallen starke Zusammenhänge zwischen den Dimensionen Alltagsweltorientierung und Autonomieunterstützung in allen Angeboten zu allen Messzeitpunkten auf. Lediglich in Angeboten zum sozialen Lernen zum zweiten Messzeitpunkt sind die Korrelationen etwas geringer und als mittlerer Effekt zu interpretieren. Ebenfalls konsistent hohe Korrelationen können zwischen Partizipation und den Anerkennungsdimensionen Individuell und Emotional berichtet werden, wobei in Medienangeboten zum zweiten Messzeitpunkt und in Leseangeboten zum dritten Messzeitpunkt die Stärke der Zusammenhänge zwischen den 
zwei Qualitätsdimensionen etwas niedriger ausfällt (vgl. Tab. 4). Ferner sind die Anerkennungsdimensionen Individuell und Emotional hoch miteinander korreliert. Zum dritten Messzeitpunkt können starke Zusammenhänge zwischen den Dimensionen Anerkennung Emotional und Autonomieunterstützung gefunden werden. Durchaus überraschend sind die Zusammenhänge zwischen kognitiver Aktivierung und emotionaler Anerkennung zum zweiten und Partizipation zum dritten Messzeitpunkt. ${ }^{\mathbf{5}}$

5 Eine genauere und detailliertere Beschreibung der Ergebnisse kann bei Sauerwein (in Vorbereitung) nachgelesen werden. 
Tab. Nummer (4): Korrelationen der Qualitätsdimensionen für jedes Ganztagsangebot zu jedem Messzeitpunkt

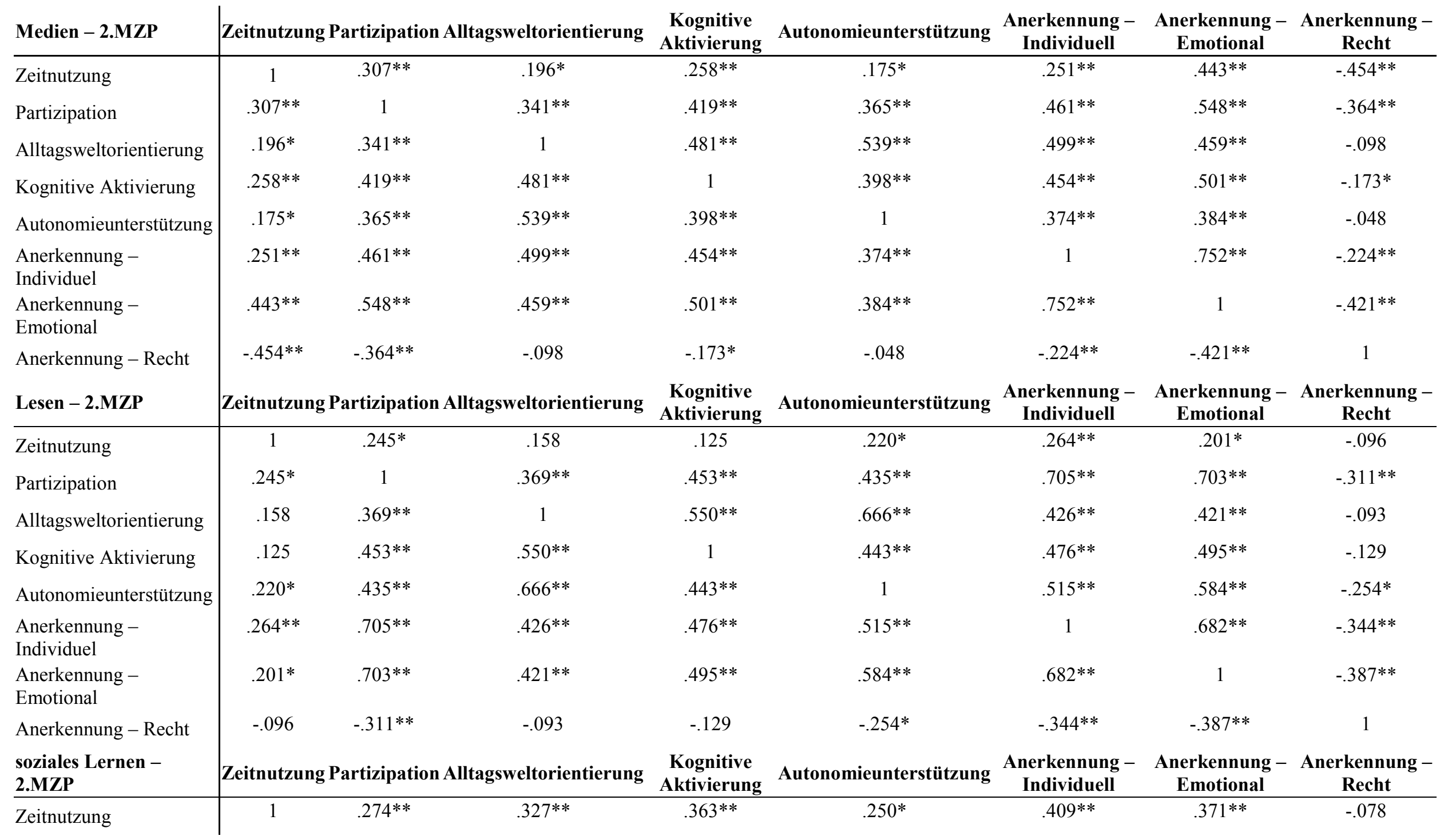




\begin{tabular}{|c|c|c|c|c|c|c|c|c|}
\hline Partizipation & $.274 * *$ & 1 & $.403 * *$ & $.672 * *$ & $.372 * *$ & $.630 * *$ & $.539 * *$ & $-.213^{*}$ \\
\hline Alltagsweltorientierung & $.327 * *$ & $.403 * *$ & 1 & $.577 * *$ & $.413^{* *}$ & $.382 * *$ & $.484 * *$ & -.109 \\
\hline Kognitive Aktivierung & $.363 * *$ & $.672 * *$ & $.577 * *$ & 1 & $.526 * *$ & $.550 * *$ & $.514^{* *}$ & -.134 \\
\hline Autonomieunterstützung & $.250 *$ & $.372 * *$ & $.413^{* *}$ & $.526^{* *}$ & 1 & $.332 * *$ & $.413 * *$ & -.035 \\
\hline $\begin{array}{l}\text { Anerkennung - } \\
\text { Individuel }\end{array}$ & $.409 * *$ & $.630 * *$ & $.382 * *$ & $.550 * *$ & $.332 * *$ & 1 & $.649 * *$ & $-.249 *$ \\
\hline $\begin{array}{l}\text { Anerkennung - } \\
\text { Emotional }\end{array}$ & $.371 * *$ & $.539 * *$ & $.484 * *$ & $.514 * *$ & $.413^{* *}$ & $.649^{* *}$ & 1 & $-.430 * *$ \\
\hline Anerkennung - Recht & -.078 & $-.213^{*}$ & -.109 & -.134 & -.035 & $-.249 *$ & $-.430 * *$ & 1 \\
\hline Medien - 3.MZP & Zeitnutzung & Partizipation & Alltagsweltorientierung & $\begin{array}{c}\text { Kognitive } \\
\text { Aktivierung } \\
\end{array}$ & Autonomieunterstützung & $\begin{array}{c}\text { Anerkennung - } \\
\text { Individuell }\end{array}$ & $\begin{array}{c}\text { Anerkennung - } \\
\text { Emotional }\end{array}$ & $\begin{array}{c}\text { Anerkennung - } \\
\text { Recht }\end{array}$ \\
\hline Zeitnutzung & 1 & $.409 * *$ & $.512 * *$ & $.375 * *$ & $.452 * *$ & $.368 * *$ & $.457 * *$ & $-.328 * *$ \\
\hline Partizipation & $.409 * *$ & 1 & $.521 * *$ & $.598 * *$ & $.593 * *$ & $.676^{* *}$ & $.691 * *$ & $-.273^{*}$ \\
\hline Alltagsweltorientierung & $.512 * *$ & $.521 * *$ & 1 & $.515 * *$ & $.584 * *$ & $.525 * *$ & $.574 * *$ & -.029 \\
\hline Kognitive Aktivierung & $.375^{* *}$ & $.598 * *$ & $.515^{* *}$ & 1 & $.466^{* *}$ & $.485^{* *}$ & $.467 * *$ & -.149 \\
\hline Autonomieunterstützung & $.452 * *$ & $.593 * *$ & $.584^{* *}$ & $.466^{* *}$ & 1 & $.453 * *$ & $.521 * *$ & -.043 \\
\hline $\begin{array}{l}\text { Anerkennung - } \\
\text { Individuel }\end{array}$ & $.368 * *$ & $.676^{* *}$ & $.525 * *$ & $.485^{* *}$ & $.453 * *$ & 1 & $.772 * *$ & -.184 \\
\hline $\begin{array}{l}\text { Anerkennung - } \\
\text { Emotional }\end{array}$ & $.457 * *$ & $.691 * *$ & $.574 * *$ & $.467 * *$ & $.521 * *$ & $.772 * *$ & 1 & -.200 \\
\hline Anerkennung - Recht & $-.328 * *$ & $-.273^{*}$ & -.029 & -.149 & -.043 & -.184 & -.200 & 1 \\
\hline Lesen - 3.MZP & Zeitnutzung & Partizipation & Alltagsweltorientierung & $\begin{array}{c}\text { Kognitive } \\
\text { Aktivierung } \\
\end{array}$ & Autonomieunterstützung & $\begin{array}{c}\text { Anerkennung - } \\
\text { Individuell }\end{array}$ & $\begin{array}{c}\text { Anerkennung - } \\
\text { Emotional }\end{array}$ & $\begin{array}{c}\text { Anerkennung - } \\
\text { Recht }\end{array}$ \\
\hline Zeitnutzung & 1 & $.550 * *$ & $.395^{* *}$ & $.362 * *$ & $.484 * *$ & $.432 * *$ & $.590 * *$ & $-.522 * *$ \\
\hline Partizipation & $.550 * *$ & 1 & $.437 * *$ & $.730 * *$ & $.460 * *$ & $.439 * *$ & $.519 * *$ & $-.367^{*}$ \\
\hline Alltagsweltorientierung & $.395^{* *}$ & $.437 * *$ & 1 & $.380 * *$ & $.652 * *$ & $.500 * *$ & $.559 * *$ & $-.393 *$ \\
\hline Kognitive Aktivierung & $.362 * *$ & $.730 * *$ & $.380^{* *}$ & 1 & $.437 * *$ & $.486^{* *}$ & $.466^{* *}$ & -.276 \\
\hline Autonomieunterstützung & $.484 * *$ & $.460 * *$ & $.652 * *$ & $.437 * *$ & 1 & $.442 * *$ & $.553 * *$ & $-.432 * *$ \\
\hline
\end{tabular}




\begin{tabular}{|c|c|c|c|c|c|c|c|c|}
\hline $\begin{array}{l}\text { Anerkennung - } \\
\text { Individuel }\end{array}$ & $.432 * *$ & $.439 * *$ & $.500 * *$ & $.486 * *$ & $.442 * *$ & 1 & $.766 * *$ & $-.436 * *$ \\
\hline $\begin{array}{l}\text { Anerkennung - } \\
\text { Emotional }\end{array}$ & $.590 * *$ & $.519 * *$ & $.559 * *$ & $.466 * *$ & $.553 * *$ & $.766 * *$ & 1 & $-.609 * *$ \\
\hline Anerkennung - Recht & $-.522 * *$ & $-.367 *$ & $-.393 *$ & -.276 & $-.432 * *$ & $-.436^{* *}$ & $-.609 * *$ & 1 \\
\hline $\begin{array}{l}\text { Soziales Lernen - } \\
\text { 3.MZP }\end{array}$ & Zeitnutzung & Partizipation & Alltagsweltorientierung & $\begin{array}{c}\text { Kognitive } \\
\text { Aktivierung }\end{array}$ & Autonomieunterstützung & $\begin{array}{c}\text { Anerkennung - } \\
\text { Individuell }\end{array}$ & $\begin{array}{c}\text { Anerkennung - } \\
\text { Emotional }\end{array}$ & $\begin{array}{c}\text { Anerkennung - } \\
\text { Recht }\end{array}$ \\
\hline Zeitnutzung & 1 & .148 & .030 & .035 & .077 & $.369^{* *}$ & .157 & $-.260 *$ \\
\hline Partizipation & .148 & 1 & $.468^{* *}$ & $.677 * *$ & $.555^{* *}$ & $.652 * *$ & $.686^{* *}$ & $-.338 * *$ \\
\hline Alltagsweltorientierung & .030 & $.468 * *$ & 1 & $.475 * *$ & $.581 * *$ & $.417 * *$ & $.488 * *$ & -.186 \\
\hline Kognitive Aktivierung & .035 & $.677 * *$ & $.475^{* *}$ & 1 & $.476^{* *}$ & $.547 * *$ & $.522 * *$ & -.096 \\
\hline Autonomieunterstützung & .077 & $.555^{* *}$ & $.581^{* *}$ & $.476^{* *}$ & 1 & $.444 * *$ & $.508 * *$ & -.171 \\
\hline $\begin{array}{l}\text { Anerkennung - } \\
\text { Individuel }\end{array}$ & $.369 * *$ & $.652 * *$ & $.417 * *$ & $.547 * *$ & $.444 * *$ & 1 & $.712 * *$ & $-.263 * *$ \\
\hline $\begin{array}{l}\text { Anerkennung - } \\
\text { Emotional }\end{array}$ & .157 & $.686^{* *}$ & $.488 * *$ & $.522 * *$ & $.508 * *$ & $.712 * *$ & 1 & $-.453 * *$ \\
\hline Anerkennung - Recht & $-.260 *$ & $-.338 * *$ & -.186 & -.096 & -.171 & $-.263 * *$ & $-.453 * *$ & 1 \\
\hline
\end{tabular}

${ }^{*} p<.05^{* *} p<.01{ }^{* * *} p<.001$ 


\section{Zusammenfassung und Diskussion der Ergebnisse}

Das Ziel dieses Beitrages besteht darin, Ganztagsangebote nicht nur als eine Ergänzung zum Schulunterricht zu konzipieren, sondern dementsprechend ein erweitertes Qualitätsverständnis vorzuschlagen, welches auch Aspekte außerschulischer Bildung beinhaltet und es empirisch zu prüfen. Die theoretisch beschriebenen Qualitätsdimensionen für Ganztagsangebote ließen sich über Schüler/innen-Fragebogen operationalisieren und empirisch trennen. Dies bestätigen konfirmatorische Faktorenanalysen sowie Korrelationen der einzelnen Qualitätsdimensionen untereinander. Die hier vorgestellten Skalen sind demnach ein geeignetes Instrument zur Erfassung von Qualität in Ganztagsangeboten aus Perspektive der Schüler/innen, welches auch in der Praxis eingesetzt werden kann.

Die Korrelationen der Angebotsqualitätsskalen zeigen, dass zwischen einzelnen Qualitätsdimensionen positive Zusammenhänge bestehen: Wird die eine Qualitätsdimension positiv bewertet, geht dies auch weitgehend mit einer positiven Bewertung einer anderen Qualitätsdimension einher. Ferner deuten die Korrelationen auf eine inhaltliche Nähe zwischen einzelnen Qualitätsdimensionen hin, auf die bereits im Theorieteil verwiesen wurde. Die Korrelationen sind jedoch nicht so stark, dass einzelne Qualitätsdimensionen zusammenzufassen wären.

Starke Zusammenhänge wurden zwischen Autonomieunterstützung, Alltagsweltorientierung und Partizipation erwartet sowie zwischen Autonomieunterstützung und den Anerkennungsdimensionen. Die empirischen Analysen können starke Zusammenhänge für Autonomieunterstützung und Alltagsweltorientierung bestätigen sowie zwischen Autonomieunterstützung und den Anerkennungsdimensionen Individuell und Emotional. Für Anerkennung Recht (Missachtung) und Partizipation können nur mittlere Zusammenhänge konstatiert werden. Bei der erlebten Partizipation kann dies u.a. auch an den Formulierungen der einzelnen Items liegen: Während bei Altagsweltorientierung, Anerkennung und Autonomieunterstützung der/ die einzelne Schüler/in adressiert wird, bezieht Partizipation die Mitsprachemöglichkeiten aller Schüler/innen mit ein. Bei der Anerkennungsdimension Recht (Missachtung) wurde nach gravierenden Formen von Missachtungserleben gefragt. Diese wurden jedoch, in sämtlichen Angeboten, von den Schüler/innen nur selten berichtet. Die Skala Partizipation hängt ebenfalls stark mit der erlebten individuellen und emotionalen Anerkennung zusammen. Einerseits ist Anerkennung eine Voraussetzung dafür, dass sich Menschen als Subjekte konstituieren und dies erscheint notwendig, um überhaupt in Gruppen (und später in der Gesellschaft) partizipieren zu können. Andererseits müssen Pädagogen davon ausgehen, dass Jugendliche bereits Fähigkeiten mitbringen, die Grundlagen sind, um gemeinsam Entscheidungen fällen zu können, die die Jugendlichen jedoch erst durch den Prozess des Partizipierens erwerben. Diese Haltung der Pädagogen kann sich positiv in erlebter Anerkennung widerspiegeln. Überraschend erscheinen die positiven Zusammenhänge zwischen kognitiver Aktivierung und emotionaler Anerkennung und Partizipation. Möglich wäre, dass aktives Partizipieren auch mit einer erhöhten kognitiven Aktivität einhergeht oder "gute Pädagogen“ Schüler/innen sowohl kognitiv ansprechen, sich gleichzeitig aber auch trauen Jugendlichen Mitspracherechte einzuräumen. Für die Zusammenhänge zwischen kognitiver Aktivierung und emotionaler Anerkennung könnte ähnlich argumentiert werden: Pädagogen, die anerkennend mit Schüler/innen umgehen, können gleichzeitig Aufgabenstellungen so formulieren, dass diese für die einzelnen Schüler/innen kognitiv aktivierend sind.

Für die Praxis bedeuten die präsentierten Ergebnisse zunächst, dass die Erweiterung der Qualitätsdimensionen für Ganztagsangebote um Aspekte der Sozialpädagogik respektive Jugendarbeit eine sinnvolle Ergänzung darstellt. Gute Ganztagsangebote zeichnen sich 
durch eine effektive Zeitnutzung, kognitiv aktivierende Aufgabenstellungen, Autonomieunterstützung, Partizipationsmöglichkeiten, eine Alltagsweltorientierte Ausrichtung sowie drei Formen von Anerkennung (emotional, individuell, rechtlich) aus. Die oben genannten Merkmale können mittels Fragebogen bei Schüler/innen erhoben werden. Weitergehende vertiefende Analysen deuten jedoch darauf hin, dass die Ausprägung der Qualität zwischen verschiedenen Ganztagsangebotskategorien - wie Medienangebote, Leseangebote oder Angebote zum sozialen Lernen - nicht verglichen werden sollte. ${ }^{6}$

Bei den präsentierten Ergebnissen und den verwendeten Daten bestehen jedoch auch Einschränkungen: Erstens ist die die Stichprobengröße für die einzelnen Angebote relativ klein und größere Stichproben könnten die Validität der Ergebnisse untermauern. Zweitens beschränken sich die Analysen auf drei verschiedene Angebotskategorien - Medien, Lesen und soziales Lernen, was letztlich der Datenlage geschuldet ist - es bleibt jedoch unklar, ob die verwendeten Qualitätsskalen auch für andere Ganztagsangebote, wie mathematische oder naturwissenschaftliche Angebote, sinnvoll eingesetzt werden können. Drittens beinhalten die verwendeten Daten nur Angaben von Schüler/innen der fünften Klasse. Zwar ist nicht davon auszugehen, dass die Analysen in einer höheren Klassenstufe zu anderen Ergebnissen führen würden, jedoch kann dies mit den vorliegenden Daten empirisch nicht geprüft werden. Zudem bleibt offen, ob die beschriebenen Qualitätsdimensionen auch an Grundschulen erhoben und empirisch voneinander unterschieden werden können. Insbesondere bei jüngeren Schüler/innen ist ungewiss, ob diese in der Lage sind, zwischen verschiedenen Qualitätsfacetten zu differenzieren. Untersuchungen für den Unterricht der Grundschule deuten jedoch darauf hin, dass, basierend auf Antworten von Schüler/innen, zwischen verschiedenen Qualitätsdimensionen differenziert werden kann (Fauth, Decristan, Riser, Klieme \& Büttner 2014 ).

Verglichen mit der Unterrichtsforschung, steckt die Forschung zu Ganztagsangeboten noch in den „Kinderschuhen“. Das hier vorgeschlagene Qualitätsmodell sollte idealerweise in weiteren Studien und aus verschiedenen Perspektiven validiert und ergänzt werden. Die vorgestellten acht Qualitätsdimensionen können sowohl für Praktiker/innen als auch für Wissenschaftler/innen einen ersten Anhaltspunkt bilden.

\section{Literatur}

Assor, A., Kaplan, H., \& Roth, G. (2002). Choice is good, but relevance is excellent: Autonomyenhancing and suppressing teacher behaviours predicting students' engagement in schoolwork. British Journal of Educational Psychology, 72, 261-278.

Balzer, N., \& Ricken, N. (2010): Anerkennung als pädagogisches Problem: Makierungen im erziehungswissenschaftlichen Diskurs. In: A. Schäfer \& C. Thompson (Eds.), Anerkennung. Paderborn; München; Wien; Zürich: Ferdinand Schöningh.

Bettmer, F. (2009). Partizipation und Anerkennung: Vorraussetzungen einer demokratischen Öffnung der Schule aus Sicht der Wissenschaft. In F. Prüss, S. Kortas, \& M. Schöpa (Eds.), Die Ganztagsschule. Von der Theorie zur Praxis ; Anforderungen und Perspektiven für Erziehungswissenschaft und Schulentwicklung (pp. 171-183). Weinheim ;, München: Juventa-Verl.

Borsboom, D. (2006): When Does Measurement Invariance Matter? In: Medical Care, 44(Suppl 3), 176. doi:10.1097/01.mlr.0000245143.08679.cc

Bundesjugendkuratorium. (2002). Zukunftsfähigkeit sichern! - Für ein neues Verhältnis von Bildung und Jugendhilfe: Eine Streitschrift des Bundesjugendkuratoriums. In R. Münchmeier, H.-U. Otto, \& U. Rabe-Kleberg (Eds.), Bildung und Lebenskompetenz. Kinder- und Jugendhilfe vor neuen Aufgaben (pp. 159-183). Opladen: Leske und Budrich.

\footnotetext{
${ }^{6}$ Siehe hierzu Sauerwein (in Vorbereitung)
} 


\section{Bundesbericht 2012/2015}

Chen, F. F. (2007): Sensitivity of Goodness of Fit Indexes to Lack of Measurement Invariance.In: Structural Equating Modeling, 14(3), 464-504.

Clausen, M. (2002). Unterrichtsqualität: eine Frage der Perspektive?: Empirische Analysen zur Übereinstimmung, Konstrukt- und Kritieriumsvalidität (Vol. 29). Münster: Waxmann.

Coelen, T. (2002): Ganztagsbildung - Ausbildung und Identitätsbildung von Kindern und Jugendlichen durch die Zusammenarbeit von Schulen und Jugendeinrichtungen. In: Neue Praxis, 32, 53-66.

Coelen, T. (2006): Ausbildung und Identitätsbildung. In: H.-U. Otto \& J. Oelkers (Eds.), Zeitgemäße Bildung. Herausforderung für Erziehungswissenschaft und Bildungspolitik: mit 9 Tabellen. München [u.a.]: Reinhardt.

Coelen, T., \& Wahner-Liesecke, I. (2009): Jugendarbeit kann auch mit (Ganztags-)Schulen wirken. In: W. Lindner (Ed.), Kinder- und Jugendarbeit wirkt. Aktuelle und ausgewählte Evaluationsergebnisse der Kinder- und Jugendarbeit (2nd ed., pp. 241-260). Wiesbaden: VS Verlag für Sozialwissenschaften.

Deci, E., \& Ryan, R. (1993): Die Selbstbestimmungstheorie der Motivation und ihre Bedeutung für die Pädagogik. In: Zeitschrift für Pädagogik, (2), 223-239.

Der Bundesminister für Jugend, F. F. u. G. (1990): Achter Jugendbericht: Bericht über Bestrebungen und Leistungen der Jugendhilfe. Bonn.

Desa, d. (2014). Evaluating Measurement Invariance of TALIS 2013 Complex Scales: Comparison between Continuous and Categorical Multiple-Group Confirmatory Factor Analyses: OECD Education Working Papers, No. 103. Retrieved from http://dx.doi.org/10.1787/5jz2kbbvlb7k-en

Ditton, H. (2002): Lehrkräfte und Unterricht aus Schülersicht. Ergebnisse einer Untersuchung im Fach Mathematik. In: Zeitschrift für Pädagogik, 48, H.2 (2002), 262-286.

Fauth, B., Decristan, J., Rieser, S., Klieme, E., \& Büttner, G. (2014). Student ratings of teaching quality in primary school: Dimensions and prediction of student outcomes. Learning and Instruction, 29, 19. doi:10.1016/j.learninstruc.2013.07.001

Fischer, N., Holtappels, H., Klieme, E., Rauschenbach, T., Stecher, L., \& Züchner, I. (2011): Ganztagsschule: Entwicklung, Qualität, Wirkungen: Längsschnittliche Befunde der Studie zur Entwicklung von Ganztagsschulen (StEG). Weinheim, Basel: Beltz Juventa.

Helmke, A., \& Klieme, E. (2008): Unterricht und Entwicklung sprachlicher Kompetenzen. In: DESIKonsortium (Ed.), Beltz Pädagogik. Unterricht und Kompetenzerwerb in Deutsch und Englisch. Ergebnisse der DESI-Studie (pp. 301-312). Weinheim, Basel: Beltz.

Helsper, W., \& Lingkost, A. (2002). Schülerpartizipation in den Antinomien der modernisierten Schulkultur. In B. Hafeneger, P. Henkenborg, \& A. Scherr (Eds.), Pädagogik der Anerkennung. Grundlagen, Konzepte, Praxisfelder. Schwalbach: Wochenschau Verlag.

Helsper, W., Böhme, J., Kramer, R.-T., \& Lingkost, A. (Eds.). (2001). Schulkultur und Schulmythos. Wiesbaden: VS Verlag für Sozialwissenschaften.

Honneth, A. (1994). Kampf um Anerkennung: Zur moralischen Grammatik sozialer Konflikte. Mit einem neuen Nachwort. Frankfurt a.M.: Suhrkamp.

Höhmann, K., Holtappels, H., \& Schnetzer, T. (2005): Ganztagsschule: Konzeption, Forschungsbefunde, aktuelle Entwicklungen. In: H. Holtappels, K. Klemm, H. Pfeifer, H.-G. Rolff, \& R. Schulz-Zander (Eds.), Jahrbuch der Schulentwicklung. Daten, Beispiele und Perspektiven (pp. 253-289). Weinheim: Juventa Verlag.

Kentler, H. (2006). Eine Theorie der Jugendarbeit - mein „Versuch 2“. In W. Lindner (Ed.), 1964 2004. Vierzig Jahre Kinder- und Jugendarbeit in Deutschland (1st ed., pp. 78-85). Wiesbaden: VS, Verl. für Sozialwiss.

Kielblock, S., Fraij, A., Hopf, A., Dippelhofer, S., \& Stecher, L. (2014): Wirkungen von Ganztagsschule auf Schüler/innen. In: T. Coelen \& L. Stecher (Eds.), Grundlagentexte Pädagogik. Die Ganztagsschule. Eine Einführung (pp. 155-174). Weinheim [u.a.]: Beltz Juventa.

Klieme, E., Lipowsky, F., Rakoczy, K., \& Ratzka, N. (2006): Qualitätsdimensionen und Wirksamkeit von Mathematikunterricht: theoretische Grundlagen und ausgewählte Ergebnisse des Projekts Pythagoras. In: M. Prenzel, \& L. Allolio-Näcke (Eds.), Untersuchungen zur Bildungsqualität von Schule. Abschlussbericht des DFG-Schwerpunktprogramms: [BIQUA] (pp. 127-146). Münster, München [u.a.]: Waxmann. 
Klieme, E., Pauli, C., \& Reusser, K. (2009): The Pythagoras study: investigating effects of teaching and learning in Swiss and German mathematics classrooms. In: T. Janik, \& T. Seidel (Eds.), The power of video studies in investigating and learning in the classroom. (pp.137-160). Münster: Waxmann.

Klieme, E., \& Rakoczy, K. (2008): Empirische Unterrichtsforschung und Fachdidaktik. Outcomeorientierte Messung und Prozessqualität des Unterrichts. Zeitschrift für Pädagogik, 54(2), 222-237.

Klieme, E., Schümer, G., \& Knoll, S. (2001): Mathematikunterricht in der Sekundarstufe I: Aufgabenstruktur und Unterrichtsgestaltung. In: E. Klieme, \& J. Baumert (Eds.), TIMMS - Impulse für Schule und Unterricht (pp. 43-58).

Kounin, J. S. (2006): Techniken der Klassenführung. Standardwerke aus Psychologie und Pädagogik, Reprints: Vol. 3. Münster [u.a.]: Waxmann.

Kunter, M. (2005): Multiple Ziele im Mathematikunterricht. Pädagogische Psychologie und Entwicklungspsychologie: Vol. 51. Münster, New York, München, Berlin: Waxmann.

Kunter, M., \& Trautwein, U. (2013): StandardWissen Lehramt: Vol. 3895. Psychologie des Unterrichts. Paderborn: Schöningh.

Mayer, R. E. (2002): Understanding Conceptual Change: A Commentary. In: M. Limón, \& L. Mason (Eds.), Reconsidering conceptual change. Issues in theory and practice (pp. 101-111). Dordrecht [u.a.]: Kluwer Academic.

Muthén, L. K., \& Muthén, B. O. (1998 - 2012). Mplus Statistical Analysis With Latent Variables: User's Guide (Seventh Edition). Los Angeles,: Muthén \& Muthén.

Muthén, B. O. \& Asparouhov, T. (2002). Latent Variable Analysis With Categorical Outcomes: Multiple-Group Groth Modeling In Mplus (Mplus Web Notes No. 4).

Müller, B., Schmidt, S., \& Schulz, M. (2005). Wahrnehmen können: Jugendarbeit und informelle Bildung. Freiburg im Breisgau: Lambertus.

Otto, H.-U., \& Rauschenbach, T. (2008): Die andere Seite der Bildung: Zum Verhältnis von formellen und informellen Bildungsprozessen (2.Auflage). Wiesbaden: VS Verlag für Sozialwissenschaften.

Prengel, A. (2006): Pädagogik der Vielfalt.: Verschiedenheit und Gleichberechtigung in Interkultureller, Feministischer und Integrativer Pädagogik (3. Auflage). Wiesbaden: VS Verlag für Sozialwissenschaften.

Prengel, A. (2013). Pädagogische Beziehungen zwischen Anerkennung, Verletzung und Ambivalenz. Leverkusen: Budrich, Barbara.

Radisch, F. (2009): Qualität und Wirkung ganztägiger Schulorganisation: Theoretische und empirische Befunde. Weinheim [u.a.]: Juventa.

Rakoczy, K. (2006): Motivationsunterstützung im Mathematikunterricht. Zur Bedeutung von Unterrichtsmerkmalen für die Wahrnehmung von Schülerinnen und Schülern. In: Zeitschrift für Pädagogik, 52(6), 822-843.

Rauschenbach, T. (2003): Kinder- und Jugendarbeit - Wege in die Zukunft. In: T. Rauschenbach, W. Düx, \& E. Sass (Eds.), Kinder- und Jugendarbeit - Wege in die Zukunft. Gesellschaftliche Entwicklungen und fachliche Herausforderungen (pp. 35-62). Weinheim: Juventa.

Rauschenbach, T. (2009): Zukunftschance Bildung: Familie Jugendhilfe und Schule in neuer Allianz. Weinheim, München: Juventa.

Rauschenbach, T., Borrmann, S., Düx, W., Liebig, R., Pothmann, J., \& Züchner, I. (2010). Lage und Zukunft der Kinder- und Jugendarbeit in Baden-Württemberg: Eine Expertise. [Stuttgart]: [Landeszentrale für Politische Bildung Baden-Württemberg].

Reinhardt, S. (2002). Jugendliche Anerkennungen zwischen Gemeinschaft und Politik: Bericht aus der Sachsen-Anhalt-Studie "Jugend und Demokratie". In B. Hafeneger, P. Henkenborg, \& A. Scherr (Eds.), Pädagogik der Anerkennung. Grundlagen, Konzepte, Praxisfelder (pp. 190-202). Schwalbach: Wochenschau Verlag.

Ricken, N. (2006): Erziehung und Anerkennung. In: Vierteljahresschrift für wissenschaftliche Pädagogik, (2), 215-230.

Sass, D. A. (2011). Testing Measurement Invariance and Comparing Latent Factor Means Within a Confirmatory Factor Analysis Framework. Journal of Psychoeducational Assessment, 29(4), 347363. doi:10.1177/0734282911406661 
Sass, D. A., Schmitt, T. A., \& Marsh, H. W. (2014). Evaluating Model Fit With Ordered Categorical Data Within a Measurement Invariance Framework: A Comparison of Estimators. Structural Equation Modeling: A Multidisciplinary Journal, 21(2), 167-180. doi:10.1080/10705511.2014.882658

Scherr, A. (2002): Subjektbildung in Anerkennungsverhältnissen: Über "soziale Subjektivität" und "gegenseitige Anerkennung" als pädagogische Grundbegriffe. In: B. Hafeneger, P. Henkenborg, \& A. Scherr (Eds.), Pädagogik der Anerkennung. Grundlagen, Konzepte, Praxisfelder (pp. 26-44). Schwalbach: Wochenschau Verlag.

Schoneville, H., \& Thole, W. (2009): Anerkennung - ein unterschätzter Begriff in der Sozialen Arbeit?: Einführung in den Schwerpunkt "Im Blickpunkt: Anerkennung". In: Soziale Passagen, (1), 133-143.

Schröder, A. (2005). Persönlichkeit und Beziehung entwickeln. In U. Deinet \& B. Sturzenhecker (Eds.), Handbuch offene Kinder- und Jugendarbeit (3rd ed., pp. 144-150). Wiesbaden: VS, Verl. für Sozialwiss.

Seidel, T. (2009): Klassenführung. In: E. Wild (Ed.), Springer-Lehrbuch. Pädagogische Psychologie (1st ed., pp. 135-148). Berlin: Springer.

Sting, S., \& Sturzenhecker, B. (2005): Bildung und Offene Kinder- und Jugendarbeit. In: U. Deinet, \& B. Sturzenhecker (Eds.), Handbuch offene Kinder- und Jugendarbeit (3rd ed., pp. 260-346). Wiesbaden: VS Verlag.

Sturzenhecker, B. (2004). Strukturbedingungen von Jugendarbeit und ihre Funktionalität für Bildung. Neue Praxis, 34(5), 444-454.

Thole, W. (2000): Kinder- und Jugendarbeit: Eine Einführung. Weinheim, München: Juventa.

Thole, W., \& Schoneville, H. (2010). Bildung und soziale Anerkennung. In U. Wilken \& W. Thole (Eds.), Kulturen der Sozialen Arbeit. Profession und Disziplin im gesellschaftlicehn Wandel (pp. 75-90). Wiesbaden: VS Verlag für Sozialwissenschaften.

Thole, W. (2013). Die Kinder- und Jugendarbeit ist ein Bildungsprojekt: Ein nochmaliges Plädoyer anlässlich der Etablierung ganztägiger Bildungslandschaften. Deutsche Jugend, 61(1), 11-16.

Vandenberg, R. J., \& Lance, C. E. (2000): A Review and Synthesis of the Measurement Invariance Literature: Suggestions, Practices, and Recommendations for Organizational Research.In: Organizational Research Methods, 3(1), 4-70.

van de Schoot, R., Lugtig, P., \& Hox, J. (2012): A checklist for testing measurement invariance. In: European Journal of Developmental Psychology, 9(4), 486-492. doi:10.1080/17405629.2012.686740

Zinser, C. (2005): Partiziption erproben und Altagswelt gestalten. In: U. Deinet, \& B. Sturzenhecker (Eds.), Handbuch offene Kinder- und Jugendarbeit (3rd ed., pp. 157-166). Wiesbaden: VS Verlag.

Zipperle, M. (2015): Jugendhilfeentwicklung und Ganztagsschule: Empirische Ergebnisse zu Herausforderungen und Chancen. Weinheim, Basel: Beltz Juventa.

\section{Markus N. Sauerwein}

Deutsches Institut für Internationale Pädagogische Forschung (DIPF) Wissenschaftlicher Mitarbeiter im Projekt Studie zur Entwicklung von Ganztagsschulen. Promoviert zum Thema Qualität in Unterricht und Angeboten in Ganztagsschulen

Arbeitsschwerpunkte: Ganztagsschulen, Qualität, Schul- und Unterrichtsforschung, Studierende der Sozialen Arbeit 